\section{TREATMENT OF GONORRHCEA.}

EFFET OF CUBEBS IN DYSPEPSIA.

\section{To the Editor of The LANCET.}

Sir,-If you deem the following cases and remarks of interest, you will spare them a corner in your invaluable periodical. I am, Sir, your obedient servant,

Corry Laughlin, M.R.C.S.,

One of the Medical Officers of the Nov. 8, 1834.

Westd. Lock IIospital, Dublin.

CASE 1.-A gentleman who was suffering under an acute attack of gonorrhoea, placed himself under my care. He had observed the discharge about one week after connexion, and shortly afterwards experienced great uneasiness in passing water, with copious increase of thick purulent matter from urethra.

Oct. 2. I directed him to take two pills composed of colocynth pill, P.D., and blue pill each gr. $\mathrm{v}$, and in the morning following an effervescing aperient draught.

Oct. 3.: Bowels comfortably freed; discharge and pain on making water the same. I prescribed $\mathrm{drachm}$ doses of eubeb pepper to be taken three times daily, and merely to apply square pieces of lint soaked in cold water under the prepuce, so as to cover the orifice of the urethra; this to be repeated frequently throughout the day.

Oct. 5. He stated that on the day following the first three doses, the pain was considerably diminished, but that the discharge was not altered. On this date, however, the uncasiness in the urethra was almost entirely gone, and the discharge mach lessened. Continue the powders and lint.

Oct. 7. Disease totally vanished.

This gentleman on taking his leave informed me that he has been a martyr to an uneasy stomach (his own words), and that it struck him that since he had taken the powders he was not quite so much affected in that respect. I asked him what kind of uneasiness he complained of, when he gave me a list of the usual symptoms of what we call dyspepsia, viz., irregular bowels, eructations, unpleasant feeling after eating but moderately, nervousness, \&c. \&c. I recommended him to try halfdrachm doses of the cubebs twice daily for a few days, and to let me see him again in a week. On his next visit his stomachic symptoms were so much relieved as to render him comparatively comfortable. I thought, however, that he had taken enough of the cubebs, and directed him to leave them off, and commence the prrtsic acid (P.D.), one drop in one ounce of distilled water three times daily. Under this remedy, now increased to two drops, this gentleman, whose appearance when I first saw him was meagre and sickly, is becoming fresh-looking and healthy.

If the symptoms above related depended upon low inflammation of the mucous surface of the stomach, it appeared to me to be probable that the cubebs must have acted in allaying it in the same manner as we know that it subdues inflammation of the mucous surface of the urethra, for I have invariably found that in cases in which this medicine shows its infiuence, the first amendment in the symptoms is relief of the pain in making water, which pain is undoubtedly caused by intense inflammation of the membrane lining this passage, and of the parts in connexion.

$C_{A S E} 2 .-A$ mate of a merchant-vessel applied to me labouring under somewhat similar gonorrhceal symptoms to those of the first case, with the addition of excoriation under the glans and prepuce, from which there was some secretion of matter.

Oct. 16. Bowels costive. Ordered gr. x Pil. Col. Comp. (P. D.) nocte. Infus. Rose క̌siss Magnes. Sulph. 3iij. Tinct. Sennce $C^{\checkmark} .5 \mathrm{ij}$; M. Mane sumendus.

17. Divide zi Piperis Cubeba in chart. oct.; 1 ter in die. Lint soaked in water.

19. No amendment whatever. Cont. medicamenta.

21. Same as 19th. Continue.

22. Ordered Bals. Copaiba కiss. Magnesia Carbonatis 3iij. Misce et divide in pil. $54 . \mathrm{j}$, 2ndis horis.

24. The pain from passing his urike nearly gone; discharge much less; and on the following day he was nearly quite well.

In the above case, and one similar in a feniale (allowing for the difference of sex) the cubebs had not the least effect; but the moment the balsam was administered the symptoms quickly diminished. I have no doubt that had I given the balsam before the cubebs, the same effect would not have been produced, as from experience $I$ find that in cases where the cubebs alone exert no influence, if followed by the balsam the disease is quickly checked.

The best and, indeed, in my opinion, the only form in which balsam copaiba ought ever to be administered is, solidified with magnesia in form of pills; it obviates all the unpleasant effects of this medicine when given in mixture. The pills are retained on the stomach in almost every case, and, moreover, it is an excellent mode of ascertaining the purity of the balsam, which, when adulterated, 
will not solidify properly with the magnesia.

I look upon the resinous ext. of copaibæ to be a useless preparation (at least in $\mathrm{my}$ experience). I never could succeed with it in a single case. I should think the virtue of the balsam is destroyed in the process of preparing it.

By the above plan of treatment, name$1 y$, using the cubebs, and, where they fail when administered alone, following them up with the balsain in the form described, and taking care of the bowels, $I$ have in ten cases out of twelve succeeded in arresting this troublesome disease in an extremely short period. I have also tried the strong injection of nitrate of silver in the early stage of gonorrhœa, often with success, but more frequently with failure, and I find that by adhering to the method $I$ have above stated (not a new one I am aware) I generally succeed.

$I$ should like to know if any of your correspondents have observed whether the cubeb pepper has had the effect of allaying stomachic derangement.

REMEDIAL POWERS OF THE

SOLUTIO HYDROIODATIS POTASSE.

By J. H. Horne, Esq., Surgeon, London.

THE hydriodate of potash mixed with cerate, and used in the form of an ointment for an external application, or a weak solution of hydriodate, for its convenient internal administration, under either form, is well known and frequently used by the profession for the cure of various diseases; but the solution of the hydriodate of potash, as an external remedy, I believe is not much known, although exceedingly efficacious in allaying excruciating pains arising from numerous neuralgic affections. The strength of the solution must be regulated accord. ing to circumstances, which will best be illustrated by detailing briefly a few cases.

CASE 1.-J. B., a strong healthy Irish labourer, in the early part of 1832 contracted a venereal discase, for the cure of which he was freely dosed by his medical attendant with mercury. He at the time of taking it was constantly exposed to the vicissitudes of the weather; however, the disease apparently yielded. At the latter part of ine same year, he was seized with pains, particularly in the large joints, which confined him to his bed. After a few days these ceased, save in the right knee-joint, which not only increased in

No. 585. pain, but enlarged to an enormous size, causing him considerable alarm, and I was therefore requested to visit him. Finding that all the joints were attacked at the commencement, I was led to form a careful diagnosis. On minutely examining the body and extremities, I found that they were covered with patches of papular eruptions, proving it to be a confirmed case of secondary syphilis, the pain of the knce arising from a specific action of the mercury on the nerves supplying that joint, which is not an unusual occurrence where mercury has been abused, or given under unfarourable circumstances. Poultices and fomentations had been used before I was consulted, without the man's deriving the least benefit from them. These I ordered to be discontinued, and directed of Hyd. Potassa $3 \mathrm{i}$, to be dissolved in $\mathrm{H}$ iss Aqua Distillate, to be applied to the knee, and the limb to be gradually straightened, to prevent hereafter a contracted joint; several instances of which deformity 1 have seen where the means employed have not had the desired effect of speedily arresting the disease. To take of Dover's Poudders $10 \mathrm{grs}$, and the same quantity of blue pill every night. The benefit was soon manifest. The pain and swelling quickly subsided, and by gradually reducing the mercury to an alterative form, the venereal taint was en. tirely destroyed, and the man perfectly recovered.

CASE 2.-A very severe case of the same kind, and highly illustrative of the great power of this form of the preparation, 1 would add, were $I$ in possession of the facts and treatment pursued while under the care of two celebrated hospital surgeons who saw it for some time, and who, failing in restoring the limb, came to the determination of amputating the leg, to which the patient refused to submit, and whose permission 1 have not been able to obtain to publish further particulars. I must therefore content myself with stating that the solution after a few days using, completely removed the pain and reduced the swelling; but from the length of time during which the disease had existed prior to the Hyd. Potassa being used, adhesions had formed, and the joint still remains contracted, though it evidently improves. In $A$ pril last the toe 3 could not be made to reach the ground by four inches. At the present time they touch it without forcing them down. In other respects he is in a perfect state of health, though when I first saw him he was almost $r$ duced to a skeleton.

CAse 3.-J.F., a waggoner, while carrying a heavy load of wheat up a high 